\title{
O Desenvolvimento de Crianças Cegas e de Crianças Videntes ${ }^{1}$ DeVtelopment of Blind Children and Sighted ChildReN
}

\author{
Maria Luiza Pontes de FRANÇA-FREITAS² \\ Maria Stella Coutinho de Alcântara GIL ${ }^{3}$
}

\begin{abstract}
RESUMO: o objetivo do presente estudo foi apresentar o desenvolvimento geral e o desenvolvimento em áreas específicas (cognição, linguagem, desenvolvimento motor, autocuidados e socialização) de uma criança cega que recebeu estimulação constante e especializada de outra criança cega que recebeu apenas estimulação assistemática e de crianças cegas com crianças videntes. Participaram deste estudo duas crianças cegas e duas crianças videntes do sexo masculino com até seis anos de idade, matriculadas em salas regulares de duas escolas públicas. O instrumento utilizado para coleta de dados foi o Inventário Portage Operacionalizado. Os dados foram analisados com base no cálculo da reta de regressão do desenvolvimento global e nas cinco áreas específicas. A partir dessas análises, verificou-se que recebendo estimulação constante e especializada a criança cega apresenta desempenhos semelhantes aos de uma criança vidente no ambiente escolar. Com base nas avaliações realizadas dos desempenhos das crianças cegas, ressalta-se que ao contrário da criança cega que recebeu estimulação constante e especializada e obteve desenvolvimento esperado para sua faixa-etária, a criança que recebeu apenas estimulação assistemática, apresentou acentuados atrasos de desenvolvimento em todas as áreas observadas. É possível supor que havendo apenas comprometimento visual, as crianças cegas devidamente estimuladas desde os primeiros anos de vida podem apresentar níveis de desenvolvimento esperados para a faixa etária. De modo geral, os resultados desse estudo, embora não permitam generalizações em função da pequena amostra, evidenciam importantes aspectos do desenvolvimento de crianças cegas e crianças videntes, contribuindo para enriquecer a literatura a respeito dessa temática.
\end{abstract}

PALAVRAS-CHAVE: Educação Especial. Desenvolvimento Infantil. Deficiências da Visão.

\begin{abstract}
The aim of this study was to present the overall development and development in specific areas (cognition, language, motor development, socialization and self-care) of a blind child who received constant and specialized stimulation with another blind child who received unsystematic stimulation and to compare blind children with sighted children. The study included two blind boys and two sighted boys up to six years old, enrolled in regular classrooms in two public schools. The instrument used for data collection was the Operationalized Portage Inventory. Data were analyzed based on the calculation of the regression line of the global development and of five specific areas. From these tests, the results indicated that the blind child who received constant and specialized stimulation during his early years presented performance similar to a sighted child in the school environment. Based on the assessments made in the performance of blind children, it was verified that unlike of blind child, who received constant and specialized stimulation and presented expected development for his age group, the other child, who received only unsystematic stimulation, presented marked developmental delays in all areas observed. One can assume that when there is only visual impairment, blind children who are properly stimulated from the earliest years of life may present developmental levels expected for their age. Even though the small sample doesn't allow for generalizations, in general the results of this study demonstrate important aspects of the development of blind children and of sighted children, contributing to enriching the literature on this topic.
\end{abstract}

KEYWORDS: Special Education. Child Development. Visual impairments

\footnotetext{
${ }^{1}$ Apoio Financeiro: CAPES - Coordenação de Aperfeiçoamento de Pessoal de Nível Superior

${ }^{2}$ Professora Doutora em Psicologia pela UFSCar e mestre em Educação Especial (PPGEES-UFSCar). mluizapf@ yahoo.com.br

${ }^{3}$ Professora Doutora vinculada ao Departamento de Psicologia da UFSCar, ao Programa de Pós-Graduação em Educação Especial e ao Programa de Pós-Graduação em Psicologia da UFSCar. stellagil@uol.com.br
} 


\section{INTRODUÇÃo}

Por muitas décadas, estudos comparativos entre crianças com desenvolvimento típico e crianças com deficiência visual consideraram que o comprometimento do sistema sensorial visual era sinônimo de dano irreparável para o desenvolvimento global destas crianças. Em uma revisão das pesquisas sobre pessoas com deficiência visual, Leme (2003) afirma que boa parte delas tem sido realizada numa perspectiva que compara o desempenho de pessoas videntes e de pessoas com deficiência visual em determinadas tarefas e cujas conclusões, com frequência, mostram atrasos ou dificuldades no desenvolvimento em detrimento das últimas. Em outra perspectiva, diversos estudos da literatura atual tem apontado semelhanças e diferenças no desenvolvimento de crianças cegas e de crianças videntes (BATISTA; ENUMO, 2000; FARIAS, 2004a; FARIAS, 2004b; NAVARRO et al., 2004; MALTA et al., 2006; SLEEUWENHOEK; BOTER; VERMEER, 1995). O embate entre a abordagem que prioriza a comparação e as abordagens que defendem a adoção do enfoque diferencial é ainda contemporâneo, mas aponta a tendência de se atentar para os fatores que produzem variação no desenvolvimento das crianças com deficiência visual sem compará-las com as crianças videntes.

Warren (1994) considera o potencial e as capacidades da criança cega e prioriza a busca da explicação das diferenças considerando exclusivamente a população de crianças cegas, levando em consideração a natureza e as causas dessas diferenças. De acordo com o autor, o enfoque diferencial permite que se ressaltem as potencialidades da criança cega, o que possibilita gerar conhecimentos que podem contribuir para propor programas de intervenção considerando as circunstâncias que otimizam o desenvolvimento delas.

Muitos estudos tem afirmado que o desenvolvimento da criança com deficiência visual segue a mesma sequência de desenvolvimento da criança vidente, apenas com a alteração do ritmo que seria mais lento (DIAS, 1995; FARIAS, 2004a). Atualmente, no entanto, considera-se que o desenvolvimento da criança com deficiência visual segue uma sequência diferente de desenvolvimento, na qual os comportamentos são aprendidos em uma ordem própria (CUNHA; ENUMO, 2003). Esses estudos não questionam mais a presença ou não de capacidades nas crianças com deficiência visual, mas destacam os aspectos nos quais se diferenciam e discutem as implicações teóricas e práticas dessas diferenças (BATISTA, 2005). De acordo com Warren (1994), as análises diferenciais mostram que, em virtualmente toda área de desenvolvimento, existem crianças deficientes visuais para as quais o progresso do desenvolvimento está pelo menos de acordo com o padrão de desenvolvimento das crianças videntes ou mesmo mostra-se frequentemente superior a esse padrão.

O desenvolvimento da criança com deficiência visual começa a se estruturar desde o nascimento a partir das habilidades de exploração, bem como das influências do seu ambiente (CUNHA; ENUMO, 2003). Cunha, Enumo e Canal (2006) destacam que uma das principais dificuldades que a criança com deficiência visual encontra em seu desenvolvimento diz respeito à lacuna na apreensão dos 
estímulos devido à ausência da percepção visual em um mundo organizado para e por pessoas com sistema sensorial visual integro. As autoras dão especial destaque ao fato de que a percepção visual é o principal meio de veiculação e de acesso às informações que serão, posteriormente, utilizadas para construção das representações da criança sobre o mundo.

Mesmo que a visão seja uma das principais fontes de acesso as informações por pessoas sem deficiência, isso não significa que a visão seja o único canal de acesso à informação (BATISTA; ENUMO, 2000). Nos primeiros anos de vida a integração sensorial, a sintetização e a interpretação das informações fornecidas por outros canais perceptivos podem e devem ser amplamente exploradas por aqueles que cuidam das crianças com deficiência visual (SOUZA et al., 2010). Existem outros recursos, além da visão, que tem um papel fundamental no desenvolvimento dessas crianças, como é o caso do sistema háptico (o tato), do sistema auditivo e do uso da linguagem entre outros.

No que diz respeito ao sistema háptico, Warren (1994) afirma que várias dimensões da percepção tátil tem sido investigadas na criança com deficiência visual. Dado o uso frequente de materiais táteis no ambiente educacional, entre outros, essas dimensões tem clara significância funcional no que diz respeito à sensibilidade tátil, à discriminação de diferentes dimensões dos objetos tais como o volume, o comprimento, largura, espessura e a forma.

Outros autores (BATISTA, 2005; FIGUEIRA, 1996; OCHAITA; ROSA, 1995) destacam diferentes contribuições do tato para o desenvolvimento da criança cega, embora reconheçam que o sistema sensorial háptico não substitui o sistema visual. De acordo com Batista (2005), o tato permite captar a temperatura, textura, forma e relações espaciais dos objetos. Esse modo particular de captação, para a autora, tem caráter sequencial e funciona a pequena distância, que corresponde ao que está ao alcance da mão, e pode ser considerado importante recurso que contribui para os processos cognitivos envolvidos na apropriação do conhecimento. $\mathrm{O}$ tato, de acordo com alguns desses autores, pode ser abordado instrumentalmente e prestar-se a processos educacionais especificamente dirigidos às crianças com deficiência visual. Desenvolver um "tato ativo" nas crianças cegas é fundamental segundo Figueira (1996) que destaca o fato de, por meio dele, as crianças cegas poderem explorar o ambiente à sua volta e formar "imagens" táteis que contribuiriam para que elas não se sentissem inseguras e desinteressadas pelo meio externo. Ochaita e Rosa (1995) definem o tato ativo, ou sistema háptico, como o sistema sensorial mais importante que a pessoa cega possui, no qual a informação é buscada de forma intencional pelo indivíduo que toca. Com isso as pessoas com deficiência visual podem passar a tomar conhecimento de detalhes mais sutis de distância e das relações espaciais facilitando sua locomoção no espaço e sua integração com o meio.

O papel e a função do sistema háptico, tal como outros sistemas perceptuais, é também objeto de controvérsias quando se analisam os processos de desenvolvimento e de aprendizagem das crianças com deficiência visual do 
ponto de vista comparativo ou compensatório, no sentido de buscar qual seria o sistema sensorial que substituiria a visão por excelência (VILLELA; GIL, 2009). Ao percorrer, portanto, os sistemas sensoriais íntegros remanescentes na criança com deficiência visual os estudiosos reconhecem a audição como outro sentido importante de reconhecimento do meio pela criança cega.

O papel da audição é destacado por Figueira (1996) para quem, na criança vidente, a audição monitorizada pelos olhos é um parâmetro do sentido de distância. De acordo com a autora, para a criança cega, a capacidade de prever a distância é um sentido subjetivo e por isso ela deve ser educada desde as primeiras horas de vida para que a localização e noção de distância adquiram objetividade. O sentido da audição, segundo a autora, também dá acesso a todos os sons que possam informar a criança a respeito das variações encontradas a sua volta e dos perigos que delas derivam. A integridade do sistema sensorial auditivo seria, portanto, uma condição importante do desenvolvimento da criança cega e, de acordo com Warren (1994), não existe evidência a respeito do funcionamento auditivo básico que indique alguma dificuldade no desenvolvimento de habilidades auditivas elementares na criança cega ou no desenvolvimento de outras habilidades que dependem de percepção auditiva.

Em se tratando do desenvolvimento cognitivo das crianças deficientes visuais a linguagem é considerada, por muitos autores, como o principal meio pelo qual a criança cega pode ter acesso ao conhecimento daquilo que não pode ver e que lhe permite comunicar-se com os outros (CUNHA; ENUMO, 2003; HALLAHAN; KAUFFMAN, 2003). A linguagem, sobretudo a fala dos primeiros anos de vida, é também a principal forma de estabelecer as interações sociais das crianças deficientes visuais, e tem um papel fundamental na mediação de todo o seu processo de aprendizagem (OLIVEIRA; MARQUES, 2005).

Muitos autores acreditam que a falta de visão não tem um efeito muito significativo na habilidade para entender e usar a linguagem (HALLAHAN; KAUFFMAN, 2003; WARREN, 1994). Warren (1994) afirma que embora a maioria das pesquisas relate que as crianças com deficiência visual falam as primeiras palavras no tempo esperado há muita variação entre elas, da mesma maneira que há diferenças individuais na aquisição da linguagem das crianças videntes. Além disso, parte dessa variação pode ser atribuída às variáveis ambientais e não propriamente à falta de visão.

Ao tratar da funcionalidade da linguagem no desenvolvimento e nos processos de aprendizagem das crianças com deficiência visual, surgem questões sobre a própria aquisição da linguagem e, portanto, sobre a aquisição dos significados das palavras pela criança com deficiência visual, bem como sobre o aspecto instrumental da linguagem na experiência da criança cega. Estas questões tem sido objeto de estudo e Warren (1994) afirma que alguns padrões de significado e uso da palavra parecem ser uma função geral da falta de experiência visual e os usos e significados específicos podem ser localizados nas próprias experiências da criança. 
O mesmo autor destaca que está claro na literatura que o vocabulário da criança com deficiência visual é fortemente fundamentado na sua própria experiência perceptiva e não simplesmente numa cópia do vocabulário das crianças videntes.

Em se tratando do desenvolvimento cognitivo das crianças com deficiência visual, há convergência dos resultados e considerações que indicam a existência de atrasos no desenvolvimento cognitivo das crianças cegas quando o parâmetro é o desenvolvimento cognitivo de crianças videntes. Ao considerar esse parâmetro, a cognição da criança deficiente visual é frequentemente considerada pobre, deficiente ou atrasada (NAVARRO; MILLÁN, 2007). Rettig (1994) ressaltou que alguns pesquisadores verificaram que as crianças apresentavam um atraso nessa área específica do desenvolvimento, principalmente em atividades que exigiam imaginação e criatividade. A análise de Cunha e Enumo (2003) coincide com a de Warren (1994) quanto a velocidade de aquisição de algumas dimensões do desenvolvimento cognitivo. Para Cunha e Enumo (2003), algumas vezes, a criança com deficiência visual pode apresentar problemas em várias áreas do desenvolvimento cognitivo, incluindo a categorização, a noção de objeto permanente e a aprendizagem. A criança com deficiência visual não tem acesso às semelhanças e diferenças entre os objetos do ambiente através do canal visual, mas pode aprender a categorizar pela indicação verbal das semelhanças ou pela percepção tátil (CUNHA et al., 2006).

Warren (1994) refere-se aos estudos nas quais foram encontradas algumas diferenças em relação ao desenvolvimento cognitivo ao comparar a criança cega com a criança com visão parcial. No que diz respeito à aquisição da permanência do objeto e conceitos de conservação, o autor afirma que se pode encontrá-los mais desenvolvidos na criança com visão parcial, entretanto, não é verificada diferença quando são consideradas outras habilidades cognitivas, como memória e atenção. Nunes e Lomônaco (2008) afirmam, ao discutir o desenvolvimento de conceitos em crianças cegas, que não é possível negar as limitações inerentes à cegueira, mas é importante perceber que muitas das dificuldades ou defasagens da criança cega devem-se muito mais à falta de informação, do que à capacidade de processá-las.

No que se refere ao desenvolvimento motor, ressalta-se que o grau da deficiência visual tem diferentes efeitos no desenvolvimento de habilidades motoras (SLEEUWENHOEK; BOTER; VERMEER, 1995). A deficiência visual, de modo geral, compromete a capacidade de locomoção e orientação da criança no espaço, requisitos fundamentais para o desempenho seguro e independente da criança no local onde se encontra. Essa deficiência afeta o desenvolvimento da percepção espacial e de distância necessárias para o bom desenvolvimento das habilidades motoras (RODRIGUES, 2002). É importante que a criança adquira essas habilidades motoras, incluindo habilidades de locomoção tanto quanto de exploração e manipulação tátil, para poder interagir efetivamente com o mundo físico (WARREN, 1994).

Em estudo realizado por Souza et al. (2010), com o intuito de avaliar o desenvolvimento neuropsicomotor de 45 crianças com e sem deficiência visual 
com idade de zero a 36 meses, foram avaliados dois grupos (um experimental e outro controle) por meio de escalas comportamentais (motora, coordenação, linguagem e social) e de visão funcional. As autoras afirmaram que o maior atraso no desenvolvimento neuropsicomotor das crianças com deficiência visual do estudo foi verificado para o comportamento de coordenação motora. Diante desse resultado percebe-se a importância da visão desde o nascimento para o desenvolvimento motor da criança.

Além da ausência da percepção visual, outros aspectos são destacados como fatores limitantes ao desenvolvimento motor de crianças com deficiência visual. De acordo com Warren (1994) o desenvolvimento mais lento nessa área em algumas crianças cegas pode ser devido à restrição de oportunidades muitas vezes agravada pela superproteção dos pais. A oportunidade, ou seja, a possibilidade de deslocamento e mobilidade é o fator mais importante na determinação das capacidades motoras de crianças com deficiência visual e a restrição de oportunidades pode gerar atrasos de desenvolvimento da locomoção. A interação da criança cega com os diferentes aspectos ambientais é, portanto, importante para a aquisição de capacidades espaciais. As crianças com deficiência visual possuem dificuldades em conhecer objetos a sua volta e em estabelecer parâmetros espaciais imprescindíveis para locomoção independente (NAVARRO et al., 2004). De acordo com Sleeuwenhoek et al. (1995), as habilidades motoras relacionadas à locomoção (engatinhar e caminhar) são significativamente mais atrasadas nas crianças cegas, talvez por causa das limitações ou ausência de feedback visual e a falta de oportunidades para imitação.

As oportunidades de imitação também são importantes para muitas das diferentes aquisições ao longo do desenvolvimento. A imitação parece um componente necessário para as habilidades que são usualmente agrupadas sob o título de autocuidado (WILLIAMS; AIELLO, 2001), que devem ser consideradas no desenvolvimento das crianças cegas. As aquisições de atividades de cuidados pessoais podem apresentar algumas diferenças por parte das crianças cegas e das videntes. As crianças cegas precisam de outros referenciais para se orientar em relação a determinadas atividades, por exemplo, pela forma como ela seleciona a roupa que irá vestir ou identifica qual a parte da frente de uma blusa. A criança vidente aprende como realizar determinadas atividades por meio da imitação, por exemplo, ela aprenderá a escovar os dentes também pela observação dos pais, irmãos e outros. No caso da criança cega, algumas vezes pode ser necessário que o adulto guie suas mãos demonstrando os movimentos implicados na escovação dos dentes ao mesmo tempo em que explica verbalmente sua ação. Dessa forma, durante seu desenvolvimento a criança deficiente visual precisa receber orientações sobre cuidados pessoais, como por exemplo, higiene pessoal, utilização da cozinha dentre outras atividades importantes para dar-lhes autonomia no dia a dia (MOTTA, 2001).

Malta et al. (2006) realizaram um estudo com o intuito de traçar o perfil funcional, ou seja, uma avaliação padronizada do desempenho da criança em atividades e tarefas típicas da rotina doméstica de crianças com deficiência visual 
com idade entre 70 e 90 meses. Essas crianças recebiam estimulação visual no Departamento de Estimulação Visual da Fundação Altino Ventura em Recife. Para realizar a avaliação foi utilizado o teste funcional "Pediatric Evaluation of Disability Inventory" (PEDI) (GISPEN, 2005) em 27 crianças: dezessete com acuidade visual normal e dez com deficiência visual. As autoras verificaram que as crianças deficientes visuais apresentaram desempenho significantemente inferior ao das crianças do grupo controle (crianças videntes com a mesma faixa-etária) na habilidade funcional de autocuidado e mobilidade.Outra área na qual pesquisas também tem encontrado particularidades em relação às crianças cegas diz respeito ao desenvolvimento social (BRAMBRING, 2001; CROCKER; ORR, 1996; D'ALLURA, 2002; ERWIN, 1993; LEME, 2003; J. OLIVEIRA, 2004; PARSONS, 1986; SANTOS, 2004; TRÖSTER; BRAMBRING, 1994). Um dos obstáculos que a criança cega encontra durante seu processo de desenvolvimento social é o fato de não responder visualmente às pessoas no ambiente. Segundo Gargiulo (2006), manter contato visual, sorrir para alguém de maneira amigável e estender a mão para falar com alguém, por exemplo, não são habilidades facilmente aprendidas pela criança que não pode ver detalhes a sua volta. Dependendo da reação dos outros com quem interage, esse obstáculo pode prejudicar ou promover suas experiências diárias com outras pessoas.

De acordo com Freitas, Del Prette e Del Prette (2007) há evidências de que as crianças com deficiência visual tem dificuldades para interagir, de formas socialmente competentes, com seus colegas e adultos, e isso não está frequentemente relacionado com a deficiência em si, mas sim com a ausência de contextos estimulantes que favoreçam tais aprendizados. Para Bruno (1993), as crianças com deficiência visual precisam ser incentivadas a utilizar os movimentos corporais, as expressões fisionômicas e gestuais como forma de comunicação pré-verbal, de imitação e representação que são elementos importantes para a socialização. A criança com deficiência visual aprenderá a imitar, cumprimentar e a brincar se encontrar pessoas disponíveis para interagir, com movimentos coativos. Esses movimentos são importantes para a compreensão da ação, permitindo o jogo imitativo de um modo diferente daquele que a criança vidente realiza. Para produzir movimentos coativos, pode-se encaixar a criança no meio do corpo do educador (pais, professor ou outros profissionais) e pelo contato físico ela compreenderá tátil-cinestesicamente os movimentos e ações realizados pelo outro.

Destaca-se que o desenvolvimento do processo das aquisições básicas da criança cega depende de fatores relacionados ao progresso da criança nas diversas áreas (FARIAS, 2004b): afetividade, cognição, linguagem, socialização, motricidade e outras. A criança precisa se desenvolver em todas as áreas e, em particular, necessita de estimulação apropriada para se locomover em segurança, com independência e agilidade, de modo a potencializar suas possibilidades de explorar dimensões físicas e sociais do meio no qual vive; algumas vezes pode necessitar da mediação do outro para estabelecer relações significativas entre coisas e eventos e, como para todas as crianças, as interações com o outro são 
a condição para todo o desenvolvimento, sobretudo, afetivo e da sociabilidade (FARIAS, 2004b; GIL; ALMEIDA, 2005; OLIVEIRA et al., 2002).

Segundo Laplane e Batista (2008) com a ausência da visão, as crianças com deficiência visual não tem o estímulo motivador das percepções visuais para seu desenvolvimento, elas não tem acesso visual aos objetos, pessoas, formas, cores e movimentos que tanto despertam curiosidade e interesse e incitam a criança a se aproximar e explorar o mundo exterior. Com essa limitação as autoras afirmam que essas crianças podem ter esse interesse diminuído pela falta de estímulos e podem, assim, tornarem-se quietas e apáticas. Nesse contexto a organização do ambiente é primordial para promover ativamente o desenvolvimento por meio dos canais sensoriais que a criança possui, possibilitando que ela seja capaz de participar nas atividades cotidianas e de aprender como qualquer criança (LAPLANE; BATISTA, 2008).

A partir dessas considerações observa-se que crianças com deficiência visual tem certas habilidades e características e encontram uma variedade de circunstâncias ambientais as quais precisam se adaptar (WARREN, 1994). É imprescindível destacar que essas circunstâncias ambientais são decisivas para o desenvolvimento da criança cega. E como a adaptação da criança varia de acordo com as oportunidades ambientais, para as crianças cegas tais oportunidades de interação com o ambiente físico e com a vida social requer, ainda mais, a intermediação do outro. É possível supor que o adulto, ao lhe propiciar um ambiente rico em estímulos promova fortemente o desenvolvimento da criança cega. A estimulação realizada de forma adequada pode contribuir para a criança cega, com deficiências adicionais ou não, adquirir senso de equilíbrio, autoconfiança e independência, tão importantes para seu desempenho geral e para aumentar as possibilidades de interação social no ambiente escolar. Acrescenta-se que se a criança cega não receber estimulação em tempo, a limitação sensorial poderá prejudicar o ganho não apenas das aquisições básicas de cognição, motricidade, linguagem e competência social (FARIAS, 2004b).

Diante do exposto o objetivo do presente estudo foi apresentar o desenvolvimento geral e o desenvolvimento em áreas específicas (cognição, linguagem, desenvolvimento motor, autocuidados e socialização) de uma criança cega que recebeu estimulação constante e especializada e de outra criança cega que recebeu apenas estimulação assistemática e de crianças cegas com crianças videntes.

\section{Método}

O estudo desenvolvido caracteriza-se como de pesquisa descritiva que tem o levantamento como delineamento. Participaram deste estudo duas crianças cegas e duas crianças videntes do sexo masculino com média de cinco anos de idade, matriculadas em salas regulares de duas escolas públicas (EMEIs), localizadas em duas cidades do interior de São Paulo. Uma das crianças cegas recebeu estimulação constante e especializada (ECE), ou seja, realizada por profissionais especializados na 
estimulação de crianças deficientes visuais e estava matriculada na sala de cinco anos. A outra criança recebeu apenas estimulação assistemática (EA), ou seja, realizada eventualmente, e estava matriculada na sala do maternal. No presente trabalho a criança cega que recebeu apenas estimulação assistemática foi designada HEA e a criança cega que recebeu estimulação constante e especializada foi designada LECE.

A criança cega HEA era um menino com cinco anos de idade com cegueira (retinopatia da prematuridade). Ele passou os primeiros meses de sua vida hospitalizado por causa da prematuridade e de pneumonias sucessivas. Com um ano e cinco meses HEA foi levado do hospital para um orfanato onde após um mês foi adotado por um casal que também tinha mais quatro filhos adotivos que apresentavam diferentes necessidades especiais. Aos três anos de idade HEA foi matriculado na Associação de Pais e Amigos dos Excepcionais (APAE) de sua cidade e frequentou essa instituição por quase um ano. No início da pesquisa já fazia um ano e seis meses que HEA havia deixado a APAE e começado a frequentar a creche (EMEI). A mãe informou que HEA frequentou uma associação para deficientes visuais em outra cidade por aproximadamente oito meses. HEA era uma criança que não interagia com os colegas e não aceitava a aproximação ou toque de outras crianças, bem como apresentava atraso na fala, comportamentos estereotipados e autoestimulatórios.

A criança cega LECE era um menino cego (catarata congênita) com cinco anos e nove meses que frequentava a sala de cinco anos (alunos com idades entre cinco e seis anos). Aos três anos de idade LECE começou a frequentar a sala de recursos em uma escola regular onde lhe ensinavam a leitura e escrita em Braille, além de outras habilidades. Aos quatro anos ainda na sala de recursos começou a frequentar a sala regular de outra escola, uma creche municipal. Frequentava também treino de futebol e natação há dois anos e uma associação que realizava estimulação, principalmente de orientação e mobilidade há um ano.

As duas crianças videntes pertenciam à sala de cada uma das crianças cegas. J era um menino três anos e seis meses e pertencia à sala da criança HEA. $\mathrm{T}$ era um menino com seis anos de idade pertencente à sala de LECE. O critério para escolha das crianças videntes foi o menor número de faltas na caderneta da professora. As mães e professoras das crianças participantes foram treinadas para serem informantes nesse estudo.

\subsection{INSTRUMENTO}

O instrumento utilizado foi o Inventário Portage Operacionalizado (WILLIAMS; AIELLO, 2001). Esse instrumento derivou do Guia Portage de Educação Pré-Escolar ("Portage Guide to Early Education" de Bluma et al., 1976) que compreende um amplo sistema de treinamento de pais e educadores.

De acordo com Williams e Aiello (2001) o Guia Portage fornece a avaliação das seguintes áreas de desenvolvimento: estimulação infantil (específica para bebês de zero a quatro meses ou para crianças com tal nível funcional) e demais áreas para 
crianças de zero a seis anos: socialização (habilidades relevantes na interação com pessoas); linguagem (comportamentos expressivos); autocuidados (independência para alimentar-se, vestir-se, banhar-se, etc); cognição (linguagem receptiva e estabelecimento de relações de semelhanças e diferenças) e desenvolvimento motor (movimentos coordenados por pequenos e grandes músculos).

Segundo Vital, Gil e Almeida (2007), a operacionalização do Inventário Portage foi realizada com o intuito de torná-lo mais útil aos projetos de intervenção e pesquisa que utilizam o Guia para realizar avaliações. As mesmas autoras afirmam que o conteúdo do Inventário Portage Operacionalizado (IPO) é uma tentativa de agrupamento de itens em termos de categorias e classes de respostas. Ainda alegam que por meio da análise torna-se possível constatar que o mesmo inclui as principais classes de respostas das melhores escalas de desenvolvimento disponíveis.

Foi necessária a adaptação de alguns itens desse inventário para possibilitar a aplicação com crianças cegas. Um exemplo de item adaptado é "escreve seu nome em Braille". Esse instrumento com os itens adaptados foi testado em uma criança cega L (catarata congênita) com cinco anos e seis meses de idade que recebeu estimulação precoce constante e especializada. Os itens adaptados para cada uma das áreas de desenvolvimento do inventário foram os seguintes: socialização (6 e 36); cognição (4, $16,22,24,27,28,29,30,33,38,39,45,53,54,58,59,60,64,67,68,75,80,81,84,91,94$, 99, 100, 101 e 107); linguagem (4, 16, 17, 20, 25, 40, 78 e 82); autocuidados (16 e 93); desenvolvimento motor $(63,73,94,95,104,109,110,117,121$ e 126).

\subsection{Procedimento de coleta de dados}

Foi realizada a aplicação do Inventário Portage Operacionalizado (WILLIAMS; AIELLO, 2001), com o intuito de avaliar o desenvolvimento das crianças cegas e das videntes nas cinco áreas específicas (socialização, linguagem, cognição, desenvolvimento motor, autocuidados) e no desenvolvimento global. Esse instrumento foi utilizado em forma de entrevista com as mães e professoras das crianças. O item era lido e a mãe ou professora informava se a criança realizava ou não o comportamento específico do item. Quando a mãe e a professora ficavam em dúvida na resposta esperavam-se alguns dias para que as informantes observassem o comportamento da criança e depois informassem se a mesma realizava ou não o comportamento questionado.

\subsection{Procedimento de ANÁlise dos dados}

Para avaliação dos resultados obtidos por meio do Inventário Portage Operacionalizado foi realizado o cálculo da reta de regressão do desenvolvimento global e nas cinco áreas do desenvolvimento. Este cálculo apresentou os totais de comportamentos estimados pelo inventário por faixa-etária e onde a criança avaliada se localizava na reta. 


\section{Resultados}

Foram avaliados os resultados em relação ao desenvolvimento global e de cada uma das cinco áreas específicas do desenvolvimento infantil (linguagem, desenvolvimento motor, autocuidados, cognição e socialização) de cada criança (HEA, J, LECE e T) nas perspectivas das mães e das professoras. Os valores apontados nas figuras de 1 a 6 mostram a frequência de comportamento alcançada pelas crianças, nas perspectivas das mães e das professoras, e a frequência de comportamentos que é esperada de acordo com o IPO (Dados do IPO).

$\mathrm{Na}$ área específica de socialização (ver Figura 1) pode ser observado que o desempenho da criança cega HEA encontra-se muito abaixo do esperado para sua faixa-etária, principalmente na perspectiva da professora. O contrário é observado com relação à criança vidente J que apresentou desempenho acima do esperado para sua faixa-etária na perspectiva da mãe e professora. Esse resultado também foi encontrado na área de linguagem, ver Figura 2. Referente à criança cega LECE, seu desempenho encontra-se próximo ao esperado para sua faixa-etária na perspectiva da mãe e da professora da mesma forma que a criança vidente $\mathrm{T}$ para a área de socialização. Na área de linguagem (ver Figura 2) apesar de a criança cega LECE ser mais nova apresentou desempenho superior ao da criança vidente $T$ tanto na perspectiva da mãe quanto da professora. Contudo ao comparar o desempenho da criança LECE com a criança HEA verifica-se uma grande discrepância tanto na área de socialização como na área de linguagem. Ao observar a Figura 3, verificase que na área de cognição as crianças HEA e J apresentaram posições contrárias no gráfico, abaixo e acima do esperado para a idade delas, respectivamente. A pontuação da criança cega LECE foi maior na perspectiva da mãe enquanto a pontuação da criança $\mathrm{T}$ foi superior na perspectiva da professora. Ao se comparar os resultados de HEA com os de LECE verifica-se diferença acentuada. HEA apresenta uma pontuação extremamente baixa na área de cognição.

Figura 1 - Desempenho por faixa-etária das crianças cegas e videntes na área específica de socialização, na avaliação do IPO respondido pelas mães e professoras

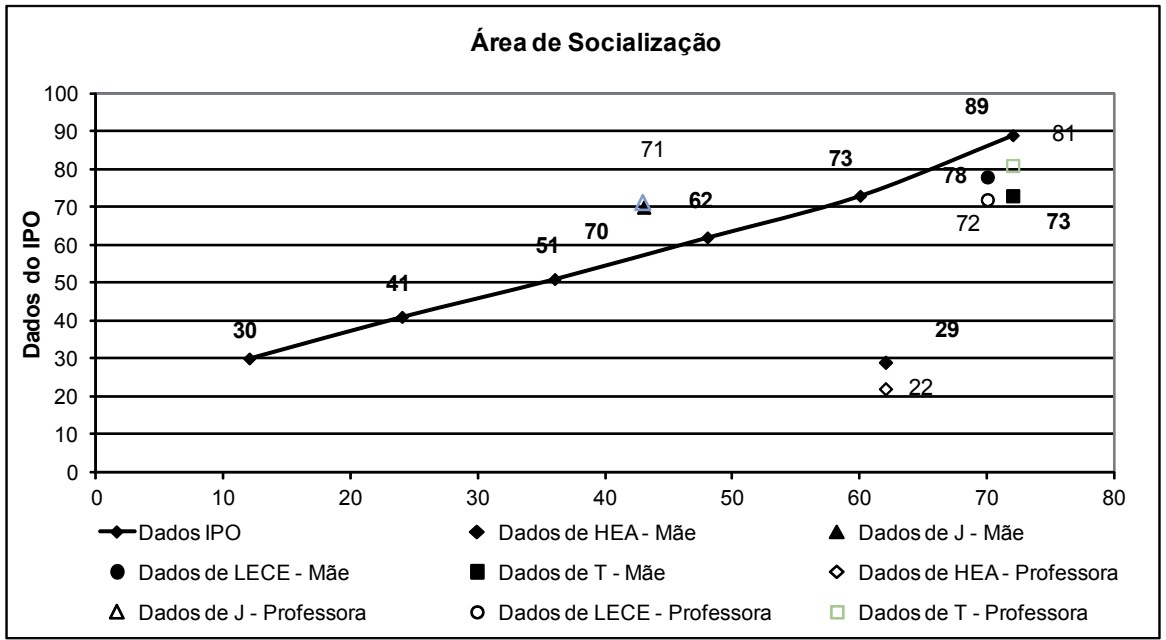


Figura 2 - Desempenho por faixa-etária das crianças cegas e videntes na área específica de linguagem, na avaliação do IPO respondido pelas mães e professoras

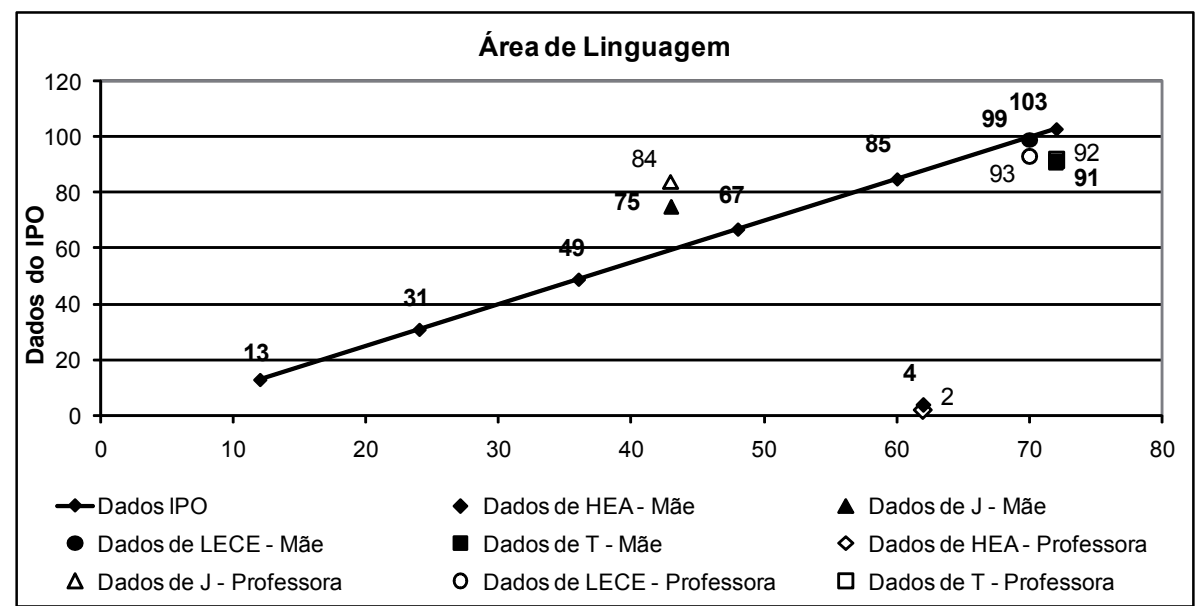

Figura 3 - Desempenho por faixa-etária das crianças cegas e videntes na área específica de cognição, na avaliação do IPO respondido pelas mães e professoras

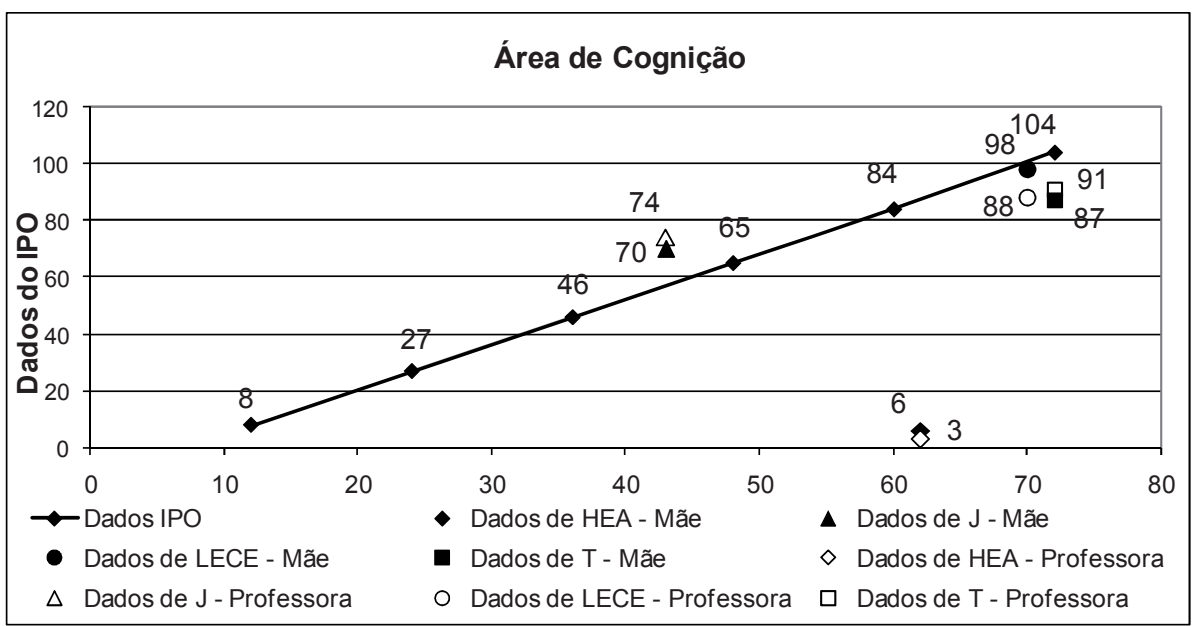


As diferenças entre HEA e LECE foram verificadas também na área específica de autocuidados como pode ser observado na Figura 4. Verifica-se, com base nessa figura, que para as crianças HEA e J a pontuação foi maior na perspectiva das mães enquanto que para as crianças LECE e T a pontuação foi superior na perspectiva da professora. Observa-se que mesmo HEA tendo uma idade superior à J seu nível de desenvolvimento foi muito abaixo do esperado para sua faixa-etária. No que diz respeito ao desenvolvimento motor, na Figura 5 verifica-se que a criança vidente J apresentou maior número de desempenhos motores do que a criança cega HEA. Referente à criança LECE, o resultado da entrevista da mãe foi diferente do resultado da entrevista com a professora. LECE pontuou mais baixo na escala na perspectiva da professora. O contrário é observado com $\mathrm{T}$ (criança vidente), que pontuou mais alto na perspectiva da professora do que na perspectiva da mãe. Verifica-se uma diferença muito grande ao se comparar o nível de desenvolvimento motor da criança cega HEA com o da criança cega LECE. A primeira encontra-se muito abaixo da pontuação esperada para a sua faixa-etária tanto na perspectiva da professora como da mãe. A segunda está próxima à pontuação esperada na perspectiva da professora e encontra-se na pontuação esperada na perspectiva da mãe. Ao observar a Figura 6 percebe-se que no desenvolvimento global foram observados os mesmos resultados alcançados nas áreas específicas. A criança HEA apresentou um nível de desempenho divergente ao da criança J e da criança LECE e esta última e a criança T apresentaram níveis de desempenhos semelhantes.

Figura 4 - Desempenho por faixa-etária das crianças cegas e videntes na área específica de autocuidados, na avaliação do IPO respondido pelas mães e professoras

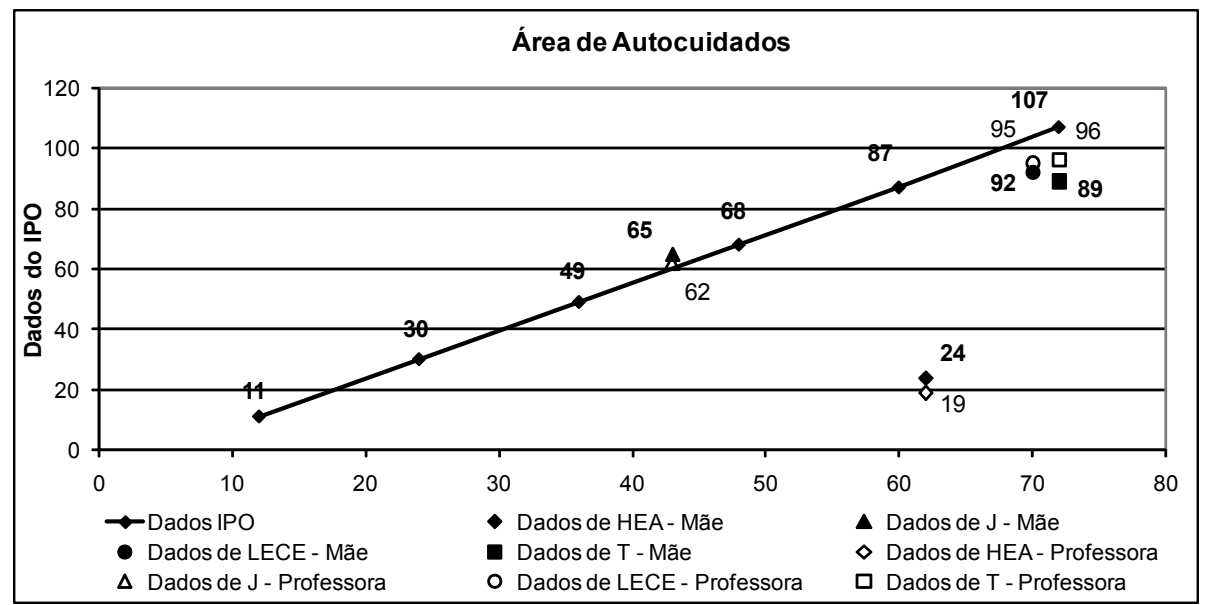


Figura 5 - Desempenho por faixa-etária das crianças cegas e videntes na área específica de desenvolvimento motor, na avaliação do IPO respondido pelas mães e professoras

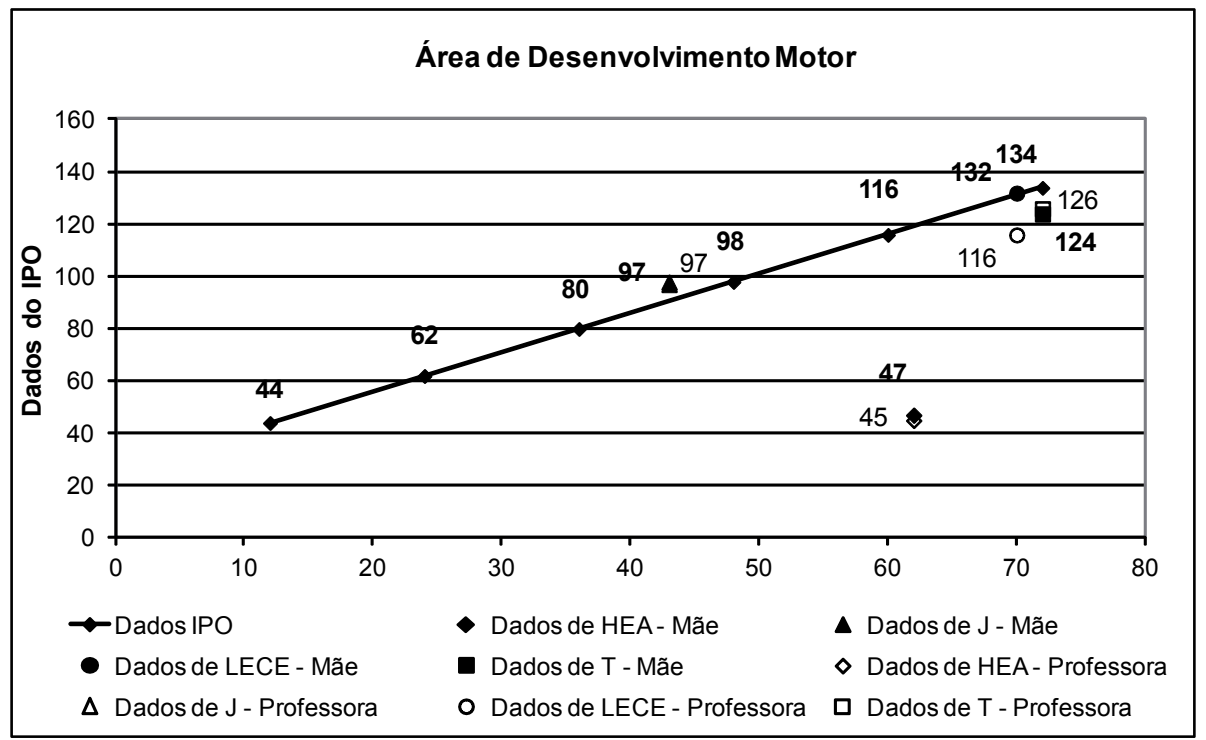

Figura 6 - Desempenho por faixa-etária das crianças cegas e videntes no desenvolvimento global, na avaliação do IPO respondido pelas mães e professoras

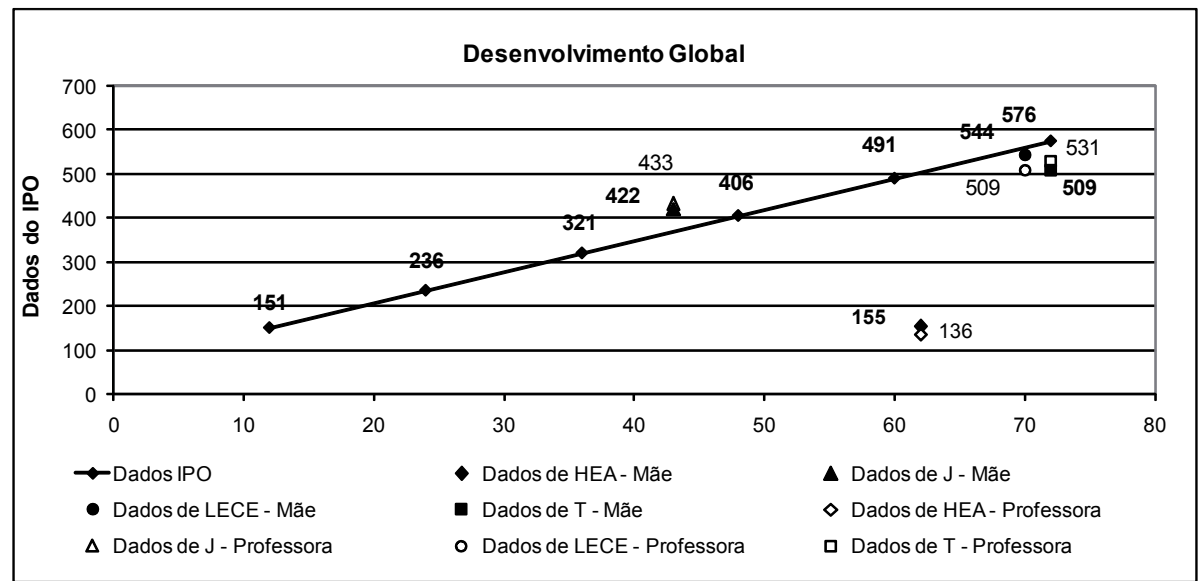

Ressalta-se que na análise do Inventário Portage Operacionalizado, apesar de HEA ser um ano e seis meses mais velho que J, ele apresentou atrasos acentuados de desenvolvimento nas cinco áreas (socialização, cognição, linguagem, autocuidados e desenvolvimento motor). Dentre essas, as áreas mais defasadas foram cognição e linguagem. O desempenho de HEA foi mais baixo na perspectiva 
da professora do que da mãe em todas as áreas. O participante $\mathrm{J}$ apresentou desempenho acima do estimado pelo Inventário para uma criança de sua faixaetária. O desempenho nas áreas de autocuidado e desenvolvimento motor foram os que mais se aproximaram da reta de regressão estimada pelo Inventário.

O desempenho de LECE na perspectiva da mãe foi igual (linguagem, cognição e desenvolvimento motor) ou um pouco abaixo (socialização e autocuidados) do estimado pelo Inventário. Na perspectiva da professora o desempenho de LECE foi um pouco abaixo da reta de regressão estimada nas cinco áreas. Os desempenhos de LECE foram mais altos na perspectiva da mãe do que na perspectiva da professora em todas as áreas com exceção da área de autocuidados. Os desempenhos de T foram um pouco abaixo do estimado pelo Inventário e dentre as cinco áreas, o desenvolvimento motor foi o mais próximo da reta de regressão. Ao contrário do que foi observado para LECE, os desempenhos de $\mathrm{T}$ foram mais baixos na perspectiva da mãe do que na perspectiva da professora. No geral, as crianças cegas tiveram pontuações maiores na perspectiva das mães enquanto as crianças videntes tiveram pontuações mais elevadas na perspectiva das professoras.

\section{Discussão}

Ao comparar os desempenhos das crianças cegas com os das crianças videntes verifica-se que o nível de desenvolvimento da criança HEA difere acentuadamente da criança J. Entretanto, ao considerar a criança LECE e a criança $\mathrm{T}$ podem ser observados desempenhos semelhantes. Com base nas avaliações realizadas nos desempenhos das crianças cegas, ressalta-se que ao contrário da criança LECE, que apresentou desenvolvimento esperado para sua faixa-etária, a criança HEA apresentou acentuados atrasos de desenvolvimento em todas as áreas observadas. Dentre essas áreas, as que HEA apresentou menor desempenho foram cognição e linguagem. $\mathrm{O}$ atraso observado no desenvolvimento de HEA pode ser resultado, dentre outros aspectos, da falta de estimulação especializada durante seus primeiros anos de vida.

De acordo com a literatura, crianças cegas que não recebem estimulação no primeiro ano de vida apresentam atrasos nas aquisições básicas de motricidade, linguagem, competência social e principalmente cognição (FARIAS, 2004a). Além disso, de acordo com Rettig (1994), crianças cegas apresentam déficits em desenvolvimento cognitivo, sobretudo em atividades que exigiam imaginação e criatividade. Todavia, o atraso nessa área específica foi observado apenas para a criança HEA e não para a criança LECE. Verifica-se, portanto, que a estimulação durante o processo de desenvolvimento da criança cega pode ocasionar diferenças em seu desempenho na área cognitiva e nas demais áreas. Atualmente, se reconhece que as crianças que não receberam estimulação no primeiro ano de vida apresentam déficits no desenvolvimento e por esse motivo necessitam de uma maior estimulação para que desenvolvam suas potencialidades. 
Ressalta-se que o fato de a criança possuir uma deficiência visual não significa que ela terá dificuldades cognitivas, emocionais e de adaptação social, mas, as formas de interação, comunicação e construção social de significados serão determinantes para o seu desenvolvimento e aprendizagem (BRASIL, 2004). A criança cega provavelmente não terá dificuldades para aprender e interagir se lhe for propiciada estimulação em tempo e um ambiente rico em experiências onde ela possa desenvolver suas capacidades.

Ao comparar as duas crianças cegas (enfoque diferencial) são consideradas suas capacidades, limitações bem como as particularidades da trajetória de desenvolvimento de cada uma, especificamente o fato de terem recebido ou não estimulação constante e especializada. Percebe-se que as variáveis ambientais contribuíram para as diferenças encontradas ao comparar o desenvolvimento de uma criança cega que recebeu estimulação constante e especializada com uma criança cega que recebeu apenas estimulação assistemática.

\section{CONCLUSÕES}

No presente estudo observou-se que recebendo estimulação constante e especializada a criança cega apresenta desempenhos semelhantes aos de uma criança vidente no ambiente escolar. É possível supor que havendo apenas comprometimento visual, as crianças cegas devidamente estimuladas desde os primeiros anos de vida podem apresentar níveis de desenvolvimento esperados para a faixa etária.

Destaca-se que a criança cega que não recebeu estimulação constante e especializada (HEA) possuía deficiências adicionais, esse fato pode gerar questões quanto à viabilidade da comparação com uma criança cega sem deficiências adicionais que recebeu esse tipo de estimulação. Entretanto, ao se adotar o enfoque diferencial, que busca explicar as diferenças dentro da própria população de crianças cegas levando em consideração a natureza e as causas dessas diferenças, torna-se relevante essa comparação.

É preciso destacar que quando a criança cega é devidamente estimulada, com profissionais qualificados e intervenções frequentes e funcionais para a vida diária, ela pode adquirir novos desempenhos e ter oportunidades de superar seus limites. Dessa forma, o curso do desenvolvimento não deve ser determinado por referência da criança vidente, pois cada criança cega tem seus limites e seu curso próprio de desenvolvimento. É preciso que os estudos estejam voltados para o que a criança cega é capaz de desempenhar e não para a ausência ou presença de visão.

De modo geral, os resultados desse estudo, embora não permitam generalizações em função da pequena amostra, evidenciam importantes aspectos do desenvolvimento de crianças cegas e crianças videntes, contribuindo para enriquecer a literatura a respeito dessa temática. 


\section{REFERENCIAS}

BATISTA, C.G. Formação de conceitos em crianças cegas: questões teóricas e implicações educacionais. Psicologia: Teoria e Pesquisa, Brasília, v.21, n.1, p.7-15, 2005.

BATISTA, C.G.; ENUMO, S.R.F. Desenvolvimento humano e impedimentos de origem orgânica: o caso da deficiência visual. In: NOVO, H.A.; MENANDRO, M.C.S. (Org.). Olhares diversos: estudando o desenvolvimento humano. Vitória: UFES, 2000. p. 157-174.

BRAMBRING, M. Integration of children with visual impairment in regular preschools. Child:Care, Health and Development, Malden, v. 27, n. 5, p. 425-438, 2001.

BRASIL, MINISTÉRIO DA EDUCAÇÃO. Saberes e práticas da inclusão: dificuldades de comunicação e sinalização. Deficiência visual - Educação Infantil. Brasília: MEC, SEESP, 2004.

BLUMA, S.M. et al., 1976. The Portage Guide to Early Education (revised edition). Portage, Wisconsin: Cooperative Educational Service Agency 12, 1976.

BRUNO, M.M.G. Desenvolvimento integral do portador de deficiência visual: da intervenção precoce à integração escolar. Rio de Janeiro: Laramara, 1993.

CROCKER, A.D.; ORR, R.R. Social behaviors of children with visual impairments enrolled in preschool programs. Exceptional Children, Fairfax, v.62, n.5, p 451-462, 1996.

CUNHA, A. C.B.; ENUMO, S.R.F. Desenvolvimento da criança com deficiência visual e interação mãe-criança: algumas considerações. Psicologia, Saúde e Doenças, Lisboa, v. 4, n. 1, p. 33-46, 2003.

CUNHA, A.C.B.; ENUMO, S.R.F.; CANAL, C.P.P. Operacionalização de escala para análise de padrão de mediação materna: um estudo com díades mãe-criança com deficiência visual. Revista Brasileira de Educação Especial, Marília, v.12, n.3, p.393-412, 2006.

D'ALLURA, T. Enhancing the social interaction skills of preschoolers with visual impairments. Journal of Visual Impairment E Blindness, Baltimore, v.96, n.9, p.577-584, 2002.

DIAS, M.E.P. Ver, Não ver e conviver. Lisboa: Secretariado Nacional de Reabilitação, 1995.

ERWIN, E.J. Social participation of young children with visual impairment in specialized and integrated environments. Journal of Visual Impairment $\mathcal{E}$ Blindness, Baltimore, v. 87, n. 5, p. 138-142, 1993.

FARIAS, G.C. Intervenção precoce: reflexões sobre o desenvolvimento da criança cega até dois anos de idade. Pensar a Prática, Goiânia, v.7, n.1, p.85-102, 2004a.

. Efeitos de um programa de intervenção precoce aplicado a uma criança cega para desenvolver suas aquisições básicas de motricidade, linguagem, competência social e cognição. Revista Benjamin Constant, Rio de Janeiro, v.29, n.3, p.9-18, 2004b.

FIGUEIRA, M.M.A. Assistência fisioterápica à criança portadora de cegueira congênita. Revista Benjamin Constant, Rio de Janeiro, v. 5, n. 4, p. 1-19, 1996.

FREITAS, M.G.; DEL PRETTE, Z.A.P.; DEL PRETTE, A. Melhorando habilidades sociais de crianças com deficiência visual: um programa de intervenção para mães. Revista Benjamin Constant, v. 13, n. 2, p. 17-27, 2007.

GARGIULO, R.M. Special education in contemporary society: an introduction to exceptionality. 2. ed. Thomson: Belmont, 2006. 
GIL, M.S.C.; ALMEIDA, N.V.F. Estudos avançados do desenvolvimento infantil. São Carlos: EdUFSCar, 2005.

GISPEN, W.H. Pediatric Evaluation of Disability Inventory (PEDI): Calibrating the Dutch version. 2005. 112p. Proefschrift (Doctoraat) - Universiteit Utrecht, Utrecht, 2005.

HALLAHAN, D.P.; KAUFFMAN, J.M. Exceptional learners: Introduction to special education. 9. ed. Boston: Allyn and Bacon, 2003.

LAPLANE, A.L.F.; BATISTA, C.G. Ver, não ver e aprender: a participação de crianças com baixa visão e cegueira na escola. Cad. Cedes, Campinas, v.28, n.75, p.209-227, 2008.

LEME, M.E.S. A representação da realidade em pessoas cegas desde o nascimento. 2003. $120 \mathrm{f}$. Dissertação (Mestrado em Educação) - Programa de Pós-Graduação em Educação Universidade de Campinas, Campinas, 2003.

MALTA, J. et al. Desempenho funcional de crianças com deficiência visual, atendidas no Departamento de Estimulação Visual da Fundação Altino Ventura. Arquivo Brasileiro de Oftalmologia, São Paulo, v. 69, n. 4, p. 571-574, 2006.

MOTTA, M.P. Atividades da vida diária: importante instrumento na habilitação do deficiente visual. Mundo Saúde, São Paulo, v. 25, n. 4, p. 358-360, 2001.

NAVARRO, A.S. et al. Coordenação motora e equilíbrio não são totalmente desenvolvidos em crianças cegas com sete anos. Arquivos de Neuropsiquiatria, São Paulo, v.62, n.3A, p.654-657, 2004.

NAVARRO, R.P.; MILLÁN, R. Inferential functioning in visually impaired children. Research in Developmental Disabilities, Baton Rouge, v.28, n.1, p.249-265, 2007.

NUNES, S.S.; LOMÔNACO, J.F.B. Desenvolvimento de conceitos em cegos congênitos: caminhos de aquisição do conhecimento. Psicologia Escolar e Educacional, Maringá, v.12, n.1, p.119-138, 2008.

OCHAITA, E.; ROSA, A. Percepção, ação e conhecimento nas crianças cegas. In: COLL, C.; PALÁCIOS, J.; MASCHESI, A. (Org.). Desenvolvimento psicológico e educação: necessidades educativas especiais e aprendizagem escolar. Porto Alegre: Artes Médicas, 1995. p.183-197.

OLIVEIRA, J.P. Análise do uso da linguagem em crianças com deficiência visual sob uma perspectiva funcional. 2004. 205f. Dissertação (Mestrado em Educação Especial) - Programa de Pós-Graduação em Educação Especial - Universidade Federal de São Carlos, São Carlos, 2004.

OLIVEIRA, J.P.; MARQUES, S.L. Análise da comunicação verbal e não-verbal de crianças com deficiência visual durante interação com a mãe. Revista Brasileira de Educação Especial, Marília, v.11, n.3, p.409-428, 2005.

OLIVEIRA, Z.M.R. et al. Creches, crianças, faz de conta E cia. 9. ed. Petrópolis: Vozes, 2002.

PARSONS, S. Function of play in low vision children (Part 1): a review of the research and literature. Journal of Visual Impairment $\mathcal{E}$ Blindness, Baltimore, v.80, n.3, p.627-630, 1986. 
RETTIG, M. The play of young children with visual impairments: characteristics and interventions. Journal of Visual Impairment $\mathcal{E}$ Blindness, Baltimore, v.88, n.9, p.410-420, 1994.

RODRIGUES, M.R.C. Estimulação precoce: a contribuição da psicomotricidade na intervenção fisioterápica como prevenção de atrasos motores na criança cega congênita nos dois primeiros anos de vida. Revista Benjamin Constant, Rio de Janeiro, v.8, n.21, p.1516, 2002.

SANTOS, F.D. A aceitação e o enfrentamento da cegueira na idade adulta. 2004, $141 \mathrm{f}$. Dissertação (Mestrado em Educação Especial) - Programa de Pós-Graduação em Educação Especial - Universidade Federal de São Carlos, São Carlos, 2004.

SLEEUWENHOEK, H.C.; BOTER, R.D.; VERMEER, A. Perceptual-motor performance and the social development of visual impaired children. Journal of Visual Impairment $\mathcal{E}$ Blindness, Baltimore, v.89, n. 4, p. 359-367, 1995.

SOUZA, T. A. et al. Descrição do desenvolvimento neuropsicomotor e visual de crianças com deficiência visual. Arq. Bras. Oftalmologia, São Paulo, v.73, n.6, p.526-530, 2010.

TRÖSTER, H.; BRAMBRING, M. The play behavior and play materials of blind and sighted infants and preschoolers. Journal of Visual Impairment $\mathcal{E}$ Blindness, Baltimore, v.88, n.9, p.421-432, 1994.

VILLELA, T.C.R.; GIL, M.S.C.A. Em busca de estratégias alternativas de ensinoaprendizagem de conceitos por crianças cegas. In: ENCONTRO IBERO AMERICANO DE EDUCAÇÃO, 4, 2009, Araraquara. Anais.... Araraquara: UNESP, 2009.

VITAL, M. L. N. V.; GIL, M. S. C. A.; ALMEIDA, N. V. F. Estimulação precoce pela brincadeira e a promoção do desenvolvimento. In: JORNADAS DE JÓVENES INVESTIGADORES DE LA AUGM: INVESTIGACIÓN, INTEGRACIÓN Y DESARROLLO, 15, 2007, Asunción. Resúmenes. Asunción: Universidad Nacional de Asunción, 2007. p. 150.

WARREN, D. H. Blindness and children: An individual differences approach. Cambridge: Cambridge University Press, 1994.

WILLIAMS, L. C. A.; AIELLO, A. L. R. O inventário portage operacionalizado: intervenção com famílias. São Paulo: Memnon, 2001.

Recebido em: 07/11/2011

Reformulado em: 30/06/2012

Aprovado em: 15/08/2012 
FRANÇA-FREITAS, M. L. P.; GIL, M. S. C. A. 